\title{
Interventricular septal hematoma complicating placement of a ventricular assist device in an infant and support with bi-atrial cannulation
}

Brian R. White, MD, PhD, ${ }^{a}$ Jill J. Savla, MD, MSCE, ${ }^{a}$ Danielle S. Burstein, MD, ${ }^{a}$

Christopher E. Mascio, MD, ${ }^{\mathrm{b}}$ Matthew J. O'Connor, MD, ${ }^{\mathrm{a}}$ Anita L. Szwast, MD, and

Lindsay S. Rogers, MD, ${ }^{a}$ Philadelphia, $\mathrm{Pa}$

From the ${ }^{\mathrm{a}}$ Division of Pediatric Cardiology, Department of Pediatrics, and ${ }^{\mathrm{b}}$ Department of Pediatric Cardiothoracic Surgery, The Children's Hospital of Philadelphia, Philadelphia, Pa.

Disclosures: Dr Mascio is a consultant for and has received honoraria from HeartWare, a VAD manufacturer not discussed in this manuscript. This manuscript discusses use of the PediMag ventricular assist device for an unapproved indication. Dr White is funded by National Heart, Lung, and Blood Institute (NHLBI) grant T32HL007915 and the Children's Hospital of Philadelphia Research Institute. Savla is funded by NHLBI grant T32HL007915. All other authors have nothing to disclose with regard to commercial support.

Received for publication Sept 12, 2019; revisions received Sept 12, 2019; accepted for publication Oct 20, 2019; available ahead of print Nov 6, 2019.

Address for reprints: Brian R. White, MD, PhD, 3401 Civic Center Blvd, 8NW, Philadelphia, PA 19104 (E-mail: whiteb1@email.chop.edu).

JTCVS Techniques 2020;1:90-2

2666-2507

Copyright (C) 2019 The Author(s). Published by Elsevier Inc. on behalf of The American Association for Thoracic Surgery. This is an open access article under the CC BY-NC-ND license (http://creativecommons.org/licenses/bync-nd/4.0/).

https://doi.org/10.1016/j.xjtc.2019.10.001

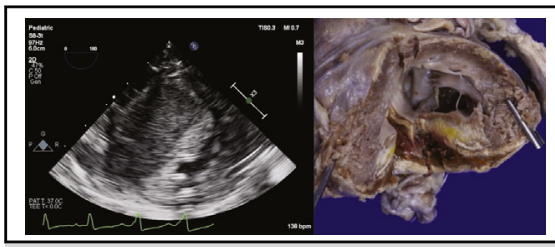

Echocardiographic and pathologic demonstration of a septal hematoma after VAD placement.

\section{CENTRAL MESSAGE}

Interventricular septal hematoma is a possible complication of VAD placement. Echocardiographers and surgeons should be alert to the possibility. Atrial cannulation is viable supportive strategy.

See Commentaries on pages 93 and 95. digoxin and was listed status 1A (by exception) for transplantation.

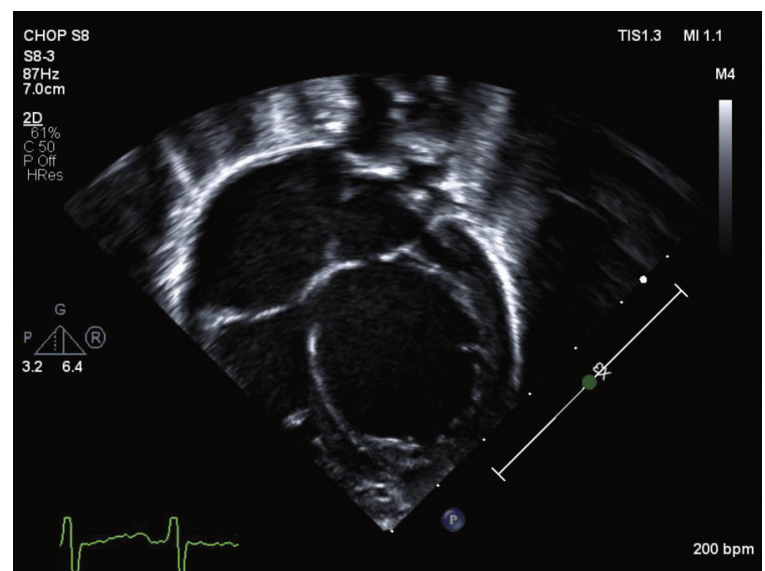

VIDEO 1. Apical 4-chamber view from a transthoracic echocardiogram performed at presentation demonstrating the diagnosis of dilated cardiomyopathy. There is severely decreased biventricular function with severe left ventricular dilation (left ventricular ejection fraction: 12\%). Video available at: https://www.jtcvs.org/article/S2666-2507(19)30001-X/fulltext. 


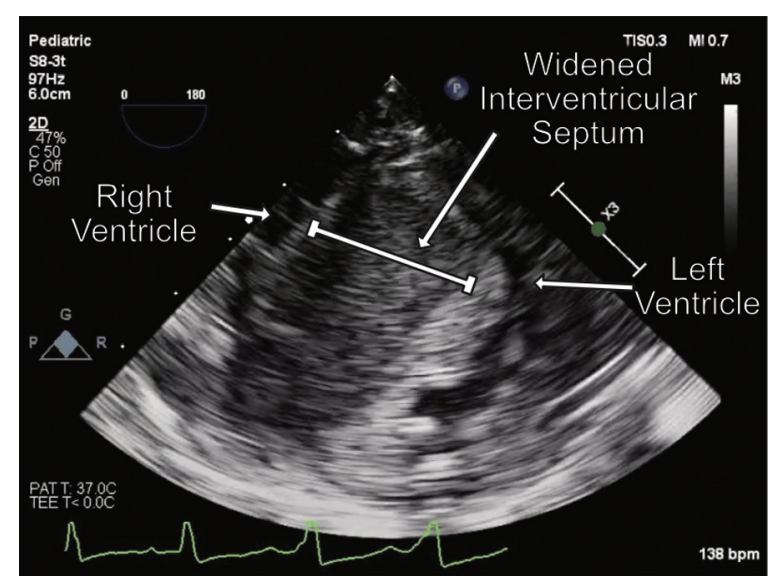

VIDEO 2. Mid-esophageal 4-chamber view from a transesophageal echocardiogram after LVAD cannulation. Note the severe septal widening with compression of both ventricles, consistent with septal hematoma. There is severely diminished septal function with moderately diminished function of the left ventricular free wall and mildly diminished function of the right ventricular free wall. The LVAD outflow cannula is visible in the left ventricular apex. $L V A D$, Left ventricular assist device. Video available at: https://www.jtcvs.org/article/S2666-2507(19)30001-X/fulltext.

Due to refractory heart failure symptoms, the patient was referred for VAD placement at 11 weeks of age. Options were limited by the patient's small size $(3.9 \mathrm{~kg})$; a temporary, paracorporeal continuous-flow left VAD (PediMag; Thoratec, Pleasanton, Calif) was chosen for left heart support. Preoperative transesophageal echocardiography (TEE) confirmed the TTE findings. A left ventricular core ventriculotomy was performed with a scalpel; LV trabeculations were divided to ensure a wider

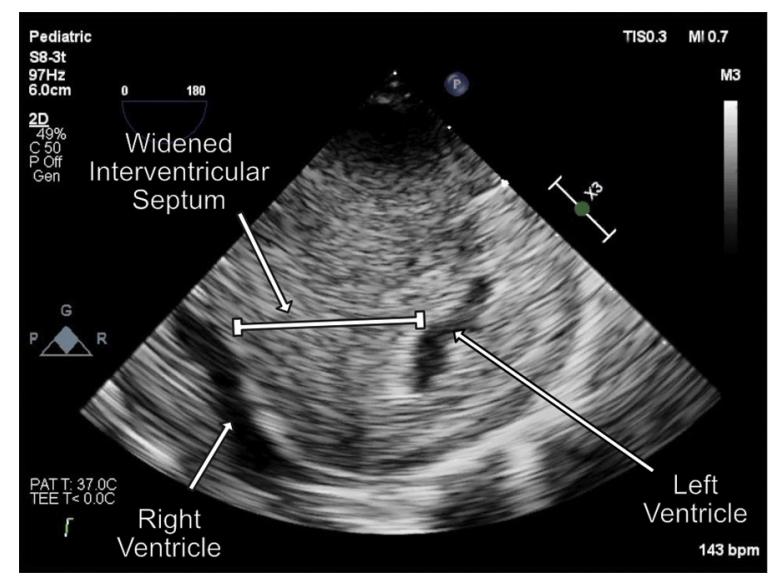

VIDEO 3. Transgastric short-axis view from a transesophageal echocardiogram at a later time after left ventricular assist device placement. The septal enlargement has increased with worsening biventricular compression. Left ventricular function is now severely diminished throughout; right ventricular free wall function is at least moderately diminished. Video available at: https://www.jtcvs.org/article/ S2666-2507(19)30001-X/fulltext.

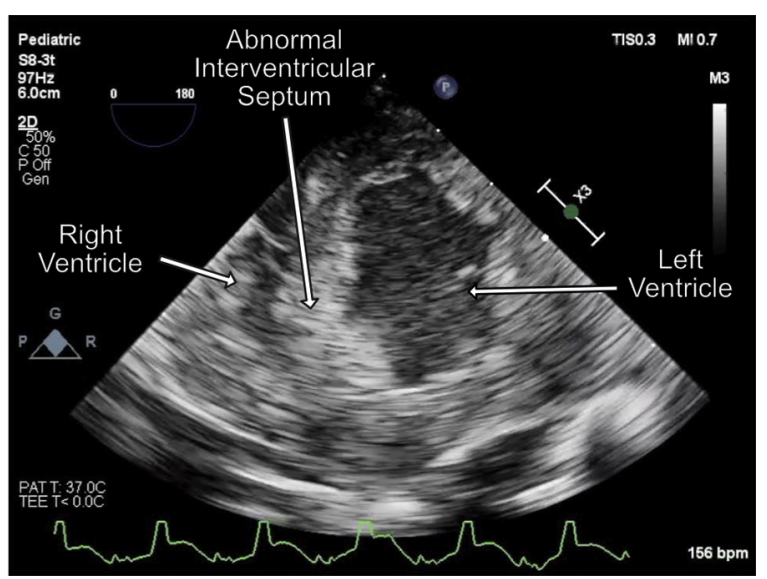

VIDEO 4. Off-axis 4-chamber sweep from a transesophageal echocardiogram performed after bi-atrial ventricular assist device cannulation. Although the interventricular septum remains abnormal, the enlargement is now less pronounced. There is continued severely diminished biventricular systolic function. Video available at: https://www.jtcvs.org/article/S2666-2507(19)30001-X/fulltext.

opening. A sewing ring was secured to the LV apex using pledgeted ETHIBOND sutures (Ethicon, Inc. Somerville, NJ), and EXCOR cannulas (Berlin Heart, Berlin, Germany) were selected. The aortic cannula was sutured to the ascending aorta using a polytetrafluoroethylene graft. VAD support was commenced.

Postoperative TEE demonstrated severe thickening of the interventricular septum with compression of both ventricular cavities and biventricular diminished systolic function (Video 2), which quickly worsened (Video 3). These findings were consistent with a septal hematoma, although

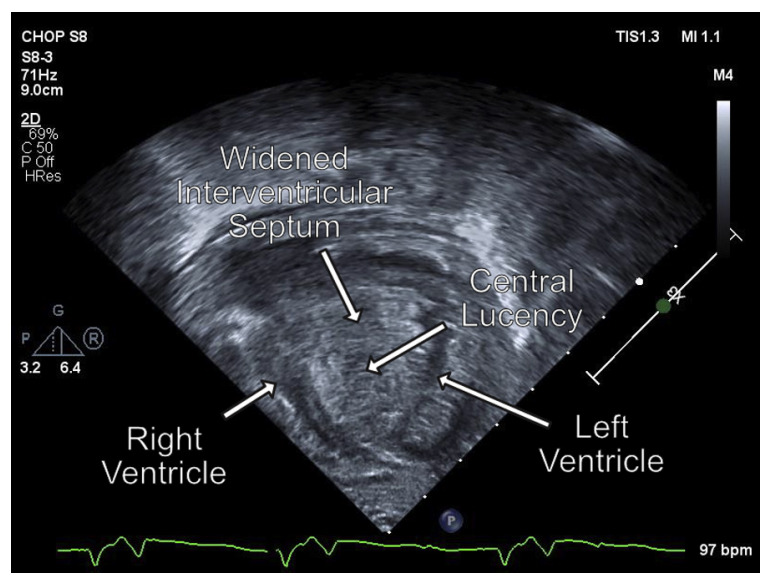

VIDEO 5. Apical 4-chamber view from a transthoracic echocardiogram performed a few days after biventricular assist device placement. The interventricular septum now has an appearance more characteristic of a septal hematoma with septal enlargement and central lucency. There is severely diminished biventricular function with minimal inflow seen through the mitral or tricuspid valves. Video available at: https://www.jtcvs.org/article/S2666-2507(19)30001-X/fulltext. 

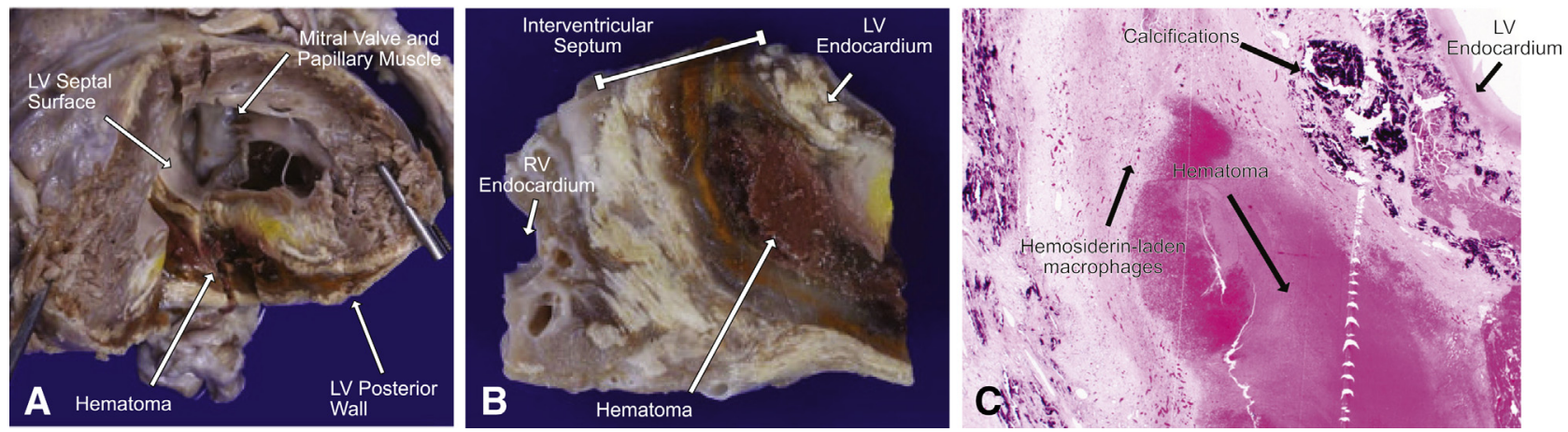

FIGURE 1. Autopsy examination of the heart demonstrating the interventricular septal hematoma. A, View from the apex with the LV opened. Hemosiderin staining is evident in the posterior LV free wall and the interventricular septum. B, Transverse section taken from the mid-portion of the interventricular septum again demonstrating the septal hematoma with surrounding fibrosis and calcification. C, Histologic examination of the interventricular septum demonstrating the hematoma with surrounding calcifications and hemosiderin-laden macrophages. $L V$, Left ventricle; $R V$, right ventricular.

atypically the septum was echo-bright and homogenous. The patient was unable to separate from bypass due to hemodynamic instability, ventricular fibrillation, and poor VAD filling. The patient was converted to biventricular VAD support via bi-atrial cannulation. The LV apical cannula was removed, and the ventriculotomy site was closed with interrupted PROLENE suture and felt strips (Ethicon). Standard 16-Fr angled bypass cannulas (Edwards Lifesciences, Irvine, Calif) were placed in the left atrial appendage and right atrial free wall. A 10-Fr straight cannula was used for the right VAD outflow in the pulmonary artery. Repeat TEE demonstrated that the interventricular septum was not as thick, but it was still abnormal (Video 4).

The patient returned to the cardiac intensive care unit. Full anticoagulation and antiplatelet therapy initially consisted of bivalirudin and aspirin. Due to thrombus in the circuits, bivalirudin was replaced with therapeutic heparin. Subsequent TTE demonstrated the typical appearance of a septal hematoma with central lucency (Video 5). The septal hematoma was managed conservatively, and despite anticoagulation and antiplatelet therapy, it did not increase in size. The patient required biventricular pump exchanges on postoperative days 4 and 24 for VAD-associated thrombi and emboli. Due to recurrent thromboembolic cerebral infarcts, advanced life support was withdrawn on postoperative day 56 .

A complete autopsy was performed, which demonstrated cardiomegaly with endocardial fibroelastosis and hypertrophy of the left ventricle. A large septal hematoma $(1.0 \times 1.2 \mathrm{~cm})$ was seen with extension into the posterior LV free wall (Figure 1). The hematoma was surrounded by fibrosis and calcification.

\section{DISCUSSION}

To our knowledge, interventricular septal hematoma complicating VAD placement has not been previously described in the literature. Although typically associated with ventricular septal defect closure, surgeons and echocardiographers need to be aware of the possibility of septal hematoma presenting in alternative situations, such as VAD placement. In our case, the hematoma was not visible on the external surface of the heart, leading to uncertainty as to the reason for poor VAD filling. Despite the unusual echocardiographic appearance, intraoperative TEE offered physiologic insight and led to implementation of an alternative surgical strategy.

The exact mechanism of hematoma formation in this case is uncertain. Although the ventricular septum was not directly manipulated during surgical intervention, in this small heart, it may have been inadvertently injured. Alternatively, the hematoma may have arisen at the site of the ventriculotomy before extending into the interventricular septum. The friable myocardium seen in neonatal cardiomyopathy may be particularly vulnerable. In infants at high risk with small ventricular cavities, atrial VAD cannulation, which has been successful in other patients in whom left ventricular filling is impaired (eg, hypertrophic or restrictive cardiomyopathy ${ }^{3-5}$ ), may be a preferable strategy.

\section{References}

1. Yoneyama F, Matsubara M, Sakamoto H, Hiramatsu Y. Interventricular septal hematoma associated with congenital heart surgery: a case report and literature review. J Thorac Cardiovasc Surg. 2017;153:e55-7.

2. Begay RL, Tharp CA, Martin A, Graw SL, Sinagra G, Miani D, et al. FLNC gene splice mutations cause dilated cardiomyopathy. J Am Coll Cardiol Basic Transl Sci. 2016;1:344-59.

3. Dykes JC, Reinhartz O, Almond CS, Yarlagadda V, Murray J, Rosenthal DN, et al. Alternative strategy for biventricular assist device in an infant with hypertrophic cardiomyopathy. Ann Thorac Surg. 2017;104:e185-6.

4. Sundararajan S, Thiruchelvam T, Hsia TY, Karimova A. New 15-mL ventricular assist device in children with restrictive physiology of the left ventricle. J Thorac Cardiovasc Surg. 2014;147:e79-80.

5. Jaquiss RD. Ventricular assistant in restrictive cardiomyopathy: making the right connection. J Thorac Cardiovasc Surg. 2016;151:e15-6. 